\title{
Super Gaussian enhancers in the Schwinger mechanism
}

\author{
Ibrahim Akal* \\ Theory Group, DESY, 22607 Hamburg, Germany
}

(Dated: December 15, 2017)

\begin{abstract}
We discuss the Schwinger mechanism in the presence of an additional uniformly oriented, weak super Gaussian of integer order $4 N+2$. Using the worldline approach, we determine the relevant critical points to compute the leading order exponential factor analytically. Already for $N=2$, we find a much stronger dynamical enhancement compared to a weak contribution of Sauter type. For higher orders, specifically for $N \rightarrow \infty$, we approach the Lorentzian case. Although such backgrounds significantly differ in Minkowski spacetime, the found coincidence applies due to identical reflection points in the instanton plane. We also treat the background in perturbation theory and show that the order parameter $N$ determines whether the weak contribution behaves perturbatively or nonperturbatively.
\end{abstract}

\section{INTRODUCTION}

The tunnelling of matter-antimatter pairs from the quantum vacuum in a background gauge field is an important nonperturbative prediction in quantum field theory [1]. For charged particles ${ }^{1}$ with mass $m$ the rate in the weakly coupled regime is exponentially suppressed below the critical field strength $E_{\mathrm{S}}=m^{2}$. Due to the extremely large value, this so-called Schwinger mechanism still could not yet be seen in the laboratory. Recently, there has also been made progress in investigating this mechanism in analogous condensed matter systems revealing interesting similarities between nonlinear quantum field theory and nonequilibrium condensed matter systems [2-7].

Temporal inhomogeneities can trigger an enormous enhancement of the tunnelling rate [8-10]. For instance, one may consider a background composed of a strong, locally static part superimposed with an additional weak but rapid alteration [11, 12]. Such composite backgrounds give rise to certain critical points [11, 13, 14] which act as reflectors in the instanton plane resulting in a drastic dynamical enhancement.

Generally, the microscopic details of the weak dependence can be very decisive. However, even alterations with a substantially distinct analytic structure in Minkowski space can lead to the same rate if the associated critical points in the instanton plane perfectly coincide [14]. Recently, such an intriguing coincidence for the leading order exponential factor has been observed between a weak Lorentzian and a super Gaussian of integer order $4 N+2$ in the limit $N \rightarrow \infty[14,15]$.

The purpose of this work is to support this insight by explicit analytic nonperturbative as well as perturbative computations. We will primarily work within the worldline formalism in quantum field theory [16, 17].

\footnotetext{
* ibrahim.akal@desy.de

1 The charge has been absorbed into the field strength. Throughout this paper we use natural units $c=1$ and $\hbar=1$.
}

\section{NONPERTURBATIVE APPROACH}

The general form for the tunnelling probability is

$$
\mathcal{P}=1-e^{-2 \Gamma}
$$

where the rate, $\Gamma$, is determined by the imaginary part of the Euler-Heisenberg effective action [18]. Due to simplifications, we focus on spin zero particles. Furthermore, we restrict ourselves to the adiabatic, nonperturbative regime and neglect contributions from the dynamical gauge field. The rate is of the form

$$
\Gamma=\mathcal{Q} e^{-\mathcal{W}_{0}}
$$

The stationary action $\mathcal{W}_{0}$ in the exponent is obtained after evaluating the worldline action

$$
\mathcal{W}=m a+i \oint d u \dot{x} \cdot \mathcal{A}\left(x_{\mu}\right)
$$

on the periodic instanton path [19] determined by

$$
m \ddot{x}_{\mu}=i a \mathcal{F}_{\mu \nu} \dot{x}_{\nu}
$$

Since the exponential factor in $\Gamma$ is the dominant quantity $[20,21]$ for the present study, we set the quantum fluctuation prefactor $\mathcal{Q}$ to unity. The kinematic invariant obeys the relation $a^{2}=\dot{x}^{2}$ due to the anti-symmetry of the field tensor $\mathcal{F}_{\mu \nu}$. We consider a purely electric background which is a uniformly oriented superposition described by

$$
\boldsymbol{E}(t)=E(f+\epsilon g) \hat{x}_{3}
$$

where $\epsilon \ll 1$ and

$$
f(t)=1, \quad g(t)=e^{-(\omega t)^{4 N+2}}, \quad N \in \mathbb{N} .
$$

In Fig. 1 the function $g$ is depicted for various $N$ including the Sauter and Lorentzian cases. 


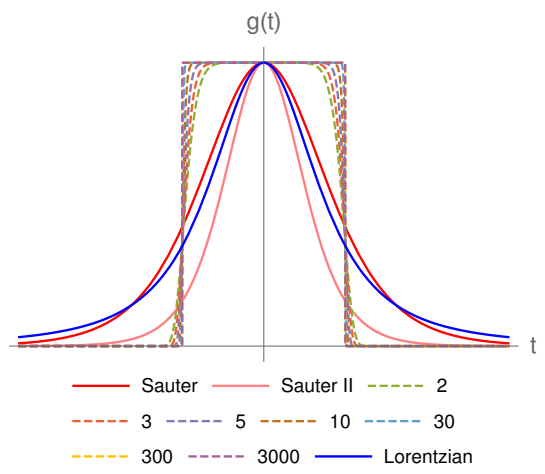

FIG. 1. Comparison of function $g$ plotted versus $t$. The numbers in the legend correspond to the integer $N$ in (6). The pink curve corresponds to a modified Sauter pulse with frequency shift $\omega \rightarrow \omega \pi / 2$ leading to the same $\mathcal{W}_{0}$ as the Lorentzian (blue). For $N \rightarrow \infty$ we approach the usual rectangular potential barrier.

After the rotation in the complex plane $\left(t \rightarrow i x_{4}\right)$, we arrive at

$$
\mathcal{A}_{3}\left(x_{4}\right)=-i E(F+\epsilon G)
$$

where

$$
\begin{aligned}
& F\left(x_{4}\right)=x_{4}, \\
& G\left(x_{4}\right)=-\frac{1}{\omega} \frac{\left(\omega x_{4}\right) \mathbf{E}_{\frac{4 N+1}{4 N+2}}\left(-\left(\omega x_{4}\right)^{4 N+2}\right)}{4 N+2} .
\end{aligned}
$$

Here, $\mathbf{E}_{n}$ denotes the exponential integral function. Inserting the vector potential (7) into the instanton equations (4), we find the following coupled system of differential equations

$$
\begin{aligned}
& \ddot{x}_{4}=+\frac{a E}{m}\left[F^{\prime}+\epsilon G^{\prime}\right] \dot{x}_{3}, \\
& \ddot{x}_{3}=-\frac{a E}{m}\left[F^{\prime}+\epsilon G^{\prime}\right] \dot{x}_{4} .
\end{aligned}
$$

The prime denotes the derivative with respect to $x_{4}$. For conventional reasons, we introduce the dimensionless combined Keldysh parameter [11]

$$
\gamma=\frac{m \omega}{E}
$$

The idea is to compute such points for which the strong contribution can be taken as negligible compared to the additional weak term. Afterwards, we can use these as effective reflection points in the instanton plane. This allows to find a sufficiently accurate expression for the stationary worldline action, for further details see [14].

Interestingly, an appropriate modification of the background shape can lead to time scale reductions in driven quantum systems, see e.g. [22]. Therefore, one may think about analogies related to such reflection points placed on the Euclidean time axis.

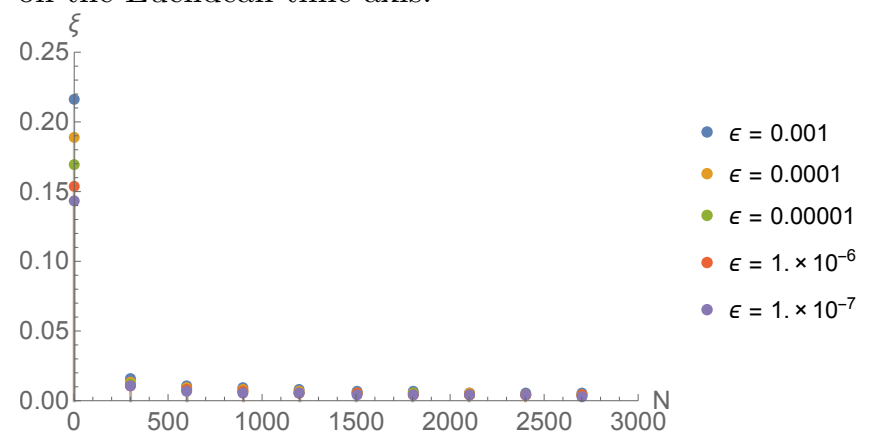

FIG. 2. Comparison of $\xi=-\mathfrak{Z} /(\alpha \mathfrak{D})$ versus $N$ (starting with $N=1$ ) for various $\epsilon$ given in the plot legend. With increasing $N$ the dependence on $\epsilon$ gets suppressed. For $N \rightarrow \infty$ we approach the Lorentzian case, i.e. $\delta=\xi \check{\gamma} \rightarrow 0($ since $\xi \rightarrow 0)$ and $\check{\gamma} \rightarrow 1$.

Proceeding in this way, we end up with the following stationary worldline action

$$
\mathcal{W}_{0} \simeq \frac{E_{\mathrm{S}}}{E} \begin{cases}\pi & \gamma<\check{\gamma} \\ 2 \check{x}_{4} \sqrt{1-\check{x}_{4}^{2}}+2 \arcsin \left(\check{x}_{4}\right) & \gamma \geq \check{\gamma}\end{cases}
$$

where

$$
\check{x}_{4}=\frac{\check{\gamma}+\delta}{\gamma+\delta}, \quad \check{\gamma}=(\ln (1 / \epsilon))^{\frac{1}{4 N+2}}, \quad \delta=-\frac{\check{\gamma}}{\alpha} \frac{\mathfrak{Z}}{\mathfrak{D}}
$$

In order to compute the remaining quantities $\alpha, \mathfrak{Z}$ and $\mathfrak{D}$ in (12), we Taylor expand the associated transcendental function in $\xi<1$, where $\delta \equiv \xi \check{\gamma}$, see App. A in [14], and truncate the resulting series after the second order which leads to the following expressions

$$
\begin{aligned}
\mathfrak{D} & :=2 \epsilon(2 N+1)\left(2 \alpha \Omega_{2}+4 \alpha N \Omega_{2}+4 N \Omega_{1}+3 \Omega_{1}\right) \\
\mathfrak{Z} & :=2 \alpha \Omega_{1} \epsilon+4 \alpha N \Omega_{1} \epsilon+4 N+\Omega \epsilon+2+\left[\left(\epsilon\left(2 \alpha \Omega_{1}+\Omega\right)+4 N\left(\alpha \Omega_{1} \epsilon+1\right)+2\right)^{2}\right. \\
& \left.-4 \alpha \epsilon(2 N+1)(4 N+\Omega \epsilon+2)\left(2 \alpha(2 N+1) \Omega_{2}+(4 N+3) \Omega_{1}\right)\right]^{1 / 2}, \\
\Omega & :=\mathbf{E}_{\frac{4 N+1}{4 N+2}}(-\alpha), \quad \Omega_{1}:=\mathbf{E}_{\frac{4 N+1}{4 N+2}-1}(-\alpha), \quad \Omega_{2}:=\mathbf{E}_{\frac{4 N+1}{4 N+2}-2}(-\alpha), \quad \alpha:=\check{\gamma}^{4 N+2} .
\end{aligned}
$$

We begin with the correction $\delta$, which we expect to vanish for increasing $N$, here expressed as $N \uparrow$. The parameter 
$\xi$ is plotted versus $N$ in Fig. 2, where the field strength ratio $\epsilon$ varies between different values as given in the plot legend. For $N=1$ the points clearly differ. However, as soon as $N \uparrow$, they rapidly merge together and converge to zero. Thus, the $\epsilon$ dependence becomes strongly suppressed and we find $\xi \rightarrow 0$, cf. Fig. 2. Remarkably, such an $\epsilon$ independence applies usually for Sauter-like pulses which have a distinct pole structure in the instanton plane, cf. e.g. [13]. Super Gaussians do not share such properties, even for very large $N$, which is therefore an interesting coincidence in itself. We will come back to this point later on.

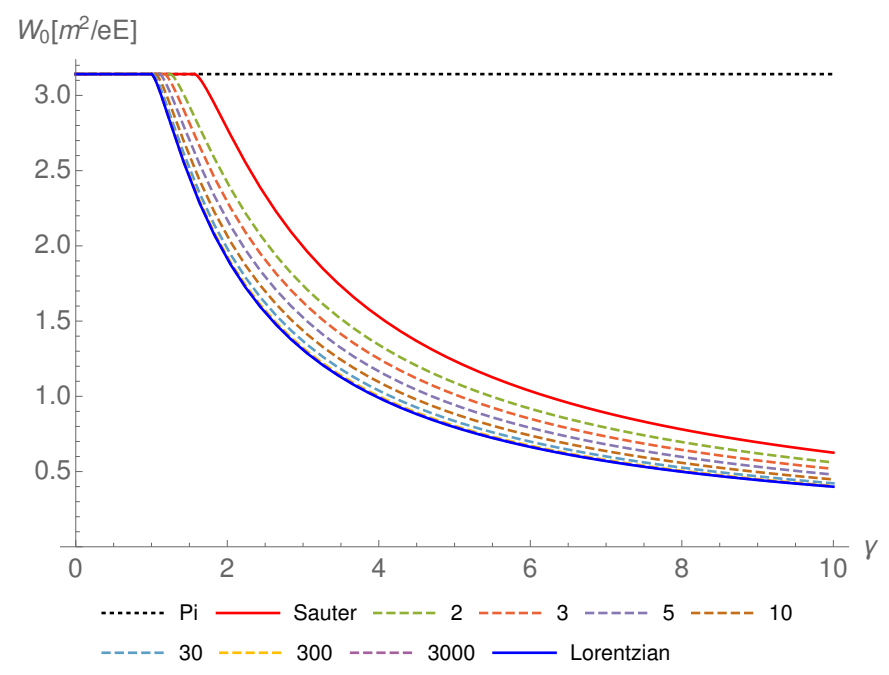

FIG. 3. Stationary worldline action $\mathcal{W}_{0}$ in units of $\left[E_{\mathrm{S}} / E\right]$. The integer values in the legend correspond to the order parameter $N$ in (6).

The nonperturbative prediction for the stationary worldline action in (11) is plotted in Fig. 3 versus $\gamma$, again for different $N$ as listed in the plot legend, including the Sauter (red solid) and Lorentzian (blue solid) case. The dashed curves depict the predictions for the super Gaussian case. Starting with $N=2$ (green), which already lies below the red solid curve, we find that as soon as $N \uparrow$ the curves converge to the blue solid one. For $N=3000$ (magenta) both results are visually indistinguishable. Furthermore, the critical threshold ${ }^{2}$, which can be approximated $^{3}$ by $\check{\gamma}$ for large $N$ quite accurately, converges to $\gamma=1$. Hence, for $N \rightarrow \infty$, corresponding to the usual rectangular potential barrier, we approach the blue solid

\footnotetext{
2 The critical threshold is assumed to be determined by the critical point where both the strong and the weak part start to contribute equally, see [14].

${ }^{3}$ For this particular type of fields the $\Delta$ correction introduced in [14] is negligible small, in particular for $N \gg 1$.
}

curve as we have also seen in direct numerical computations ${ }^{4}$. The numerically found threshold matches with our prediction $\check{\gamma}$. We conclude that for order parameters

$$
N \in \mathbb{N}_{>1}
$$

the corresponding curves for $\mathcal{W}_{0}$ lie within the throat-like region bounded by the red (Sauter) and blue (Lorentzian) one, cf. Fig. 3.

\section{PERTURBATIVE EXPANSION}

For weak Sauter-like pulses the first order contribution in perturbation theory respective $\epsilon$ turns out to be sufficient to reproduce the leading order exponential factor in $\mathcal{P}$. Since the super Gaussian in the limit $N \rightarrow \infty$ behaves as the Lorentzian, it is reasonable to expect such a coincidence on perturbative level as well. The orderby-order contributions in $\epsilon$ can be obtained on basis of the Fourier transform $\widetilde{g}$, see e.g. [23]. The Lorentzian in Fourier space reads

$$
\widetilde{g}(\varpi)=\frac{\varpi}{\omega} \mathbf{K}_{1}\left(\frac{\varpi}{\omega}\right)
$$

with $\mathbf{K}_{1}$ being the first-order modified Bessel function of the second kind. For super Gaussians of the form (6), the representation in Fourier space is much more difficult to obtain. However, for the present discussion we may follow a slightly different approach. We construct the super Gaussian $\left(\mathrm{SG}_{4 N+2}\right)$, mainly in the (almost) rectangular potential barrier limit, i.e. $N \gg 1$, which is the interesting case here, via the convolution of an ordinary Gaussian,

$$
\mathrm{G}_{\sigma_{g}} \hat{=} e^{-\left(t / \sigma_{g}\right)^{2}},
$$

with the standard rectangular function,

$$
\mathrm{R}_{\sigma_{r}} \hat{=} \operatorname{rect}\left(\frac{t}{2 \sigma_{r}}\right)
$$

Thus, in order to compute $\widetilde{g}$, we proceed according to the following prescription

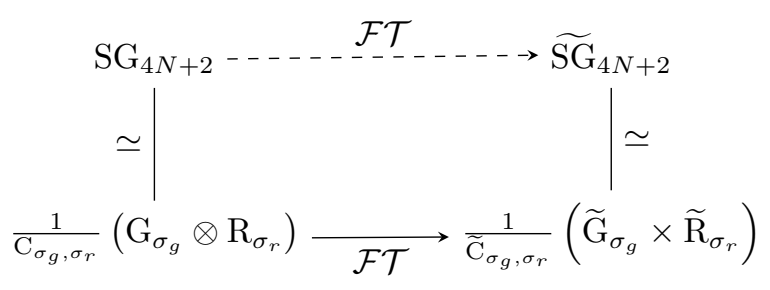

\footnotetext{
4 The accuracy of the analytical prediction in (11) increases as soon as $N \uparrow$. A similar behaviour applies for $\epsilon \downarrow$ with moderate $N$ as discussed in [14] for $N=\{0,1\}$.
} 
where $\otimes$ denotes the convolution product and $\mathrm{C}_{\sigma_{g}, \sigma_{r}}, \widetilde{\mathrm{C}}_{\sigma_{g}, \sigma_{r}}$ are some normalisation factors. Identifying

$$
\begin{aligned}
N & \leftrightarrow 1 / \kappa, \\
\sigma_{r} & \leftrightarrow 1 / \omega,
\end{aligned}
$$

with $\kappa:=\sigma_{g} / \sigma_{r}$, we finally write

$$
\widetilde{g}(\varpi)=\frac{\omega}{\varpi} \sin \left(\frac{\varpi}{\omega}\right) \exp \left(-\frac{\kappa^{2} \varpi^{2}}{4 \omega^{2}}\right)
$$

imposing the condition $\kappa \ll 1$.

\section{A. First order in $\epsilon$}

Following the discussion in [23], the general expression after perturbing the interaction Hamiltonian in the Furry picture gives

$$
\mathcal{P}=V_{3} \int \frac{d p^{3}}{(2 \pi)^{3}}\left|\ldots+\epsilon \int \frac{d \varpi}{2 \pi} \widetilde{g} \Pi_{p}+\ldots\right|^{2} .
$$

For simplifications we assume $\boldsymbol{p}=0$ which is reasonable, since the spectrum for backgrounds considered here is symmetrically peaked around the origin. Then the matrix element at $\mathcal{O}(\epsilon)$ takes the form

$$
\Pi_{0}(\varpi)=e^{\frac{E_{S}}{E}\left(\left[\frac{\varpi}{2 m} \sqrt{1-\left(\frac{\varpi}{2 m}\right)^{2}}+\arcsin \left(\frac{\varpi}{2 m}\right)\right]-\frac{\pi}{2}\right)}
$$

which, not surprisingly, becomes unsuppressed for $\varpi=$ $2 m$. In order to perform a saddle point approximation to the $\varpi$ integral in (20), we assume $\varpi \gg \omega$ such that for the Lorentzian this results in

$$
\widetilde{g} \simeq \exp \left(-\frac{\varpi}{\omega}\right)
$$

The corresponding saddle point is

$$
\varpi_{\mathrm{sp}}=2 m \sqrt{1-1 / \gamma^{2}}
$$

leading to the previously introduced threshold $\gamma \geq 1$. For $\gamma=1$ the contribution $\widetilde{g}\left(\varpi_{\mathrm{sp}}\right)$ is maximal where the exponential $\Pi_{0}\left(\varpi_{\mathrm{sp}}\right)$ approaches its minimum. Setting $x:=\varpi / \omega$, we find the following integral solution

$$
\int_{0}^{\infty} d x x \mathbf{K}_{1}(x)=\frac{\pi}{2}
$$

where the integrand follows from (15). Note that the approximate expression (22) obeys

$$
\int_{0}^{\infty} d x \exp (-x)=\frac{2}{\pi} \int_{0}^{\infty} d x x \mathbf{K}_{1}(x)
$$

For the super Gaussian the interesting limit we want to focus on is $\kappa \rightarrow 0$. In this case we cannot write an exponential expression for $\widetilde{g}$ assuming $\varpi \gg \omega$. However,

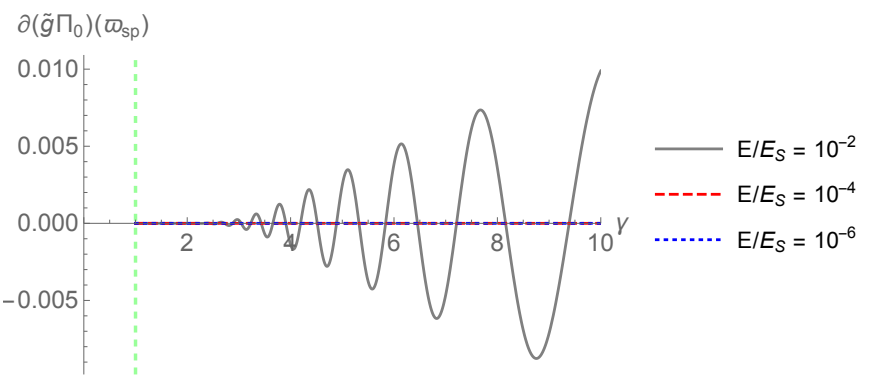

FIG. 4. Saddle point condition (26) evaluated in $\varpi_{\mathrm{sp}}$ for different ratios $E / E_{\mathrm{S}}$ plotted versus $\gamma$. The vertical dashed line is placed at the critical threshold $\gamma=1$.

according to the findings in Sec. II we set $\varpi=\varpi_{\mathrm{sp}}$, see (23), and check whether this solves the saddle point condition

$$
\left.\partial\left(\widetilde{g} \Pi_{0}\right)\right|_{\kappa \rightarrow 0}=0
$$

where $\partial \equiv \partial / \partial_{\varpi}$. It turns out that for the nonperturbative weak field regime, i.e. $E / E_{\mathrm{S}} \ll 1$ and $\omega \ll m$, the condition (26) is fulfilled, cf. Fig. 4. For $E / E_{\mathrm{S}}=10^{-2}$ and $\gamma \gtrsim 2$ the curve becomes increasingly oscillating until it settles down at $\simeq 0.15$. Such a breakdown is reasonable, since according to $2 E / E_{\mathrm{S}}=\omega / \mathrm{m}$ the gray solid curve with $\omega / m>2 \times 10^{-2}$ almost approaches the Compton scale. An approximate validity condition for $\varpi_{\mathrm{sp}}$ can be therefore given as

$$
\gamma E / E_{\mathrm{S}} \lesssim 10^{-2}
$$

which is obviously satisfied for $E / E_{\mathrm{S}}=10^{-4}$ (red, dashed) and $E / E_{\mathrm{S}}=10^{-6}$ (blue, dotted) depicted in Fig. 4.

Now, applying again the previous definition $x$ to (19), we obtain in the relevant limit the same integral solution as in the Lorentzian case, cf. Eq. (24),

$$
\int_{0}^{\infty} d x \frac{\sin (x)}{x} e^{-\kappa^{2} x^{2} / 4} \rightarrow \frac{\pi}{2}
$$

For large $x$ the integrand oscillates around the function in Eq. (15), but asymptotically converges to zero. Therefore, since $\varpi_{\text {sp }}$ works for any $\omega$, at least for $\omega \ll m$, we may conclude that the threshold at $\gamma=1$ applies for the super Gaussian in the limit $N \rightarrow \infty$ as well. This is exactly what we have found in our previous nonperturbative approach, see Sec. II, which has been also confirmed in direct numerical computations. Note that, as soon as $\kappa$ is taken to be sufficiently large, the latter coincidence will not apply anymore.

For completeness, let us briefly discuss the Sauter case for which we find

$$
\widetilde{g}(\varpi)=\frac{\varpi}{\omega} \operatorname{csch}\left(\frac{\pi}{2} \frac{\varpi}{\omega}\right) .
$$

Again, for $\varpi \gg \omega$ we can write an approximate expression as

$$
\widetilde{g} \simeq \exp \left(-\frac{\pi}{2} \frac{\varpi}{\omega}\right)
$$


leading to the known critical threshold $\gamma \geq \pi / 2$. Remarkably, integrating the function (29) leads to

$$
\int_{0}^{\infty} d x x \operatorname{csch}\left(\frac{\pi}{2} x\right)=1
$$

which equals to (24) divided by $\pi / 2$. We can again relate the approximate integrand to the exact one via

$$
\int_{0}^{\infty} d x \exp \left(-\frac{\pi}{2} x\right)=\frac{2}{\pi} \int_{0}^{\infty} d x x \operatorname{csch}\left(\frac{\pi}{2} x\right)
$$

which is analogous to the relation in (25) obtained in the weak Lorentzian case.

The findings above suggest that the integral

$$
\int_{0}^{\infty} d x \widetilde{g}(\omega x)
$$

seems to incorporate useful information about the impact of the additional weak dependence. Namely, if (33) coincides for two different setups, such as a Lorentzian and a super Gaussian with $N \rightarrow \infty$ or a Sauter pulse with frequency shift $\omega \rightarrow \omega \pi / 2$ [14], respectively, see Fig. 1, the stationary action $\mathcal{W}_{0}$ turns out to be equal as well.

This is an interesting observation, since although such backgrounds crucially differ in Minkowski space, one obtains the same tunnelling exponential. On the other hand, we may take these insights as a strong evidence that such a dynamical enhancement is mainly determined by the (effective) reflection points in the instanton plane which, in contrast, do perfectly agree.

\section{B. Higher orders in $\epsilon$}

For higher order contributions we rely on the general expansion

$$
\mathcal{P} \simeq \mathcal{P}_{0}+\epsilon \mathcal{P}_{1}+\epsilon^{2} \mathcal{P}_{2}+\mathcal{O}\left(\epsilon^{3}\right) .
$$

The zeroth order term stems again only from the strong background dependence. The functions $\mathcal{P}_{\mathfrak{N}}$ can be obtained on basis of the $\mathfrak{N}$ photon master formula in a static background, see e.g. [17]. Performing a saddle point approximation with respect to the proper and worldline time, see Eq. (5.5) in [23], the leading order contribution reads

$$
\begin{aligned}
& \mathcal{P}_{\mathfrak{N}} \simeq \int d \varpi_{1} \widetilde{g}\left(\varpi_{1}\right) \ldots \int d \varpi_{\mathfrak{N}} \widetilde{g}\left(\varpi_{\mathfrak{N}}\right) \\
& \times \exp \left(\frac{2 m^{2}}{E}\left[\Sigma \sqrt{1-\Sigma^{2}}+\arcsin (\Sigma)-\frac{\pi}{2}\right]\right)
\end{aligned}
$$

where $0<\Sigma<1$ is defined as

$$
\Sigma:=\frac{1}{2 m} \sum_{i=1}^{J} \varpi_{i}
$$

and

$$
\sum_{l \in\{1, \ldots, J, \ldots, \mathfrak{N}\}} \varpi_{l}=2 m \Sigma+\sum_{j=J+1}^{\mathfrak{N}} \varpi_{j}=0
$$

applies due to energy conservation. Note that the exponential in (35) is of the same form as in (21). Without loss of generality let us assume $2 m \Sigma \gg \omega$. So for the Lorentzian we use again the approximate expression (22) and compute the $\varpi_{l}$ integrals via (37). Carrying out a saddle point approximation with respect to $\Sigma$ results in

$$
\begin{aligned}
& \mathcal{P}_{\mathfrak{N}} \simeq \exp \left(-\frac{4 m^{2}}{E} \frac{\Sigma_{\mathrm{sp}}}{\gamma}\right) \\
& \times \exp \left(\frac{2 m^{2}}{E}\left[\Sigma_{\mathrm{sp}} \sqrt{1-\Sigma_{\mathrm{sp}}^{2}}+\arcsin \left(\Sigma_{\mathrm{sp}}\right)-\frac{\pi}{2}\right]\right)
\end{aligned}
$$

where $\Sigma_{\mathrm{sp}}=\sqrt{1-1 / \gamma^{2}}$.

For the super Gaussian in the rectangular potential barrier limit, i.e. $\kappa \rightarrow 0$, the situation is not much different. First, we solve the $\varpi_{l}$ integrals using condition (37). The prefactor in front of the exponential in (35) takes the form

$$
\prod_{i} \frac{\omega}{\varpi_{i}} \sin \left(\frac{\varpi_{i}}{\omega}\right) \prod_{j} \frac{\omega}{\varpi_{j}} \sin \left(\frac{\varpi_{j}}{\omega}\right)
$$

with

$$
\begin{aligned}
& \varpi_{i}=\frac{2 m \Sigma}{J-1}, \quad i \in\{2, \ldots, J\} \\
& \varpi_{j}=\frac{-2 m \Sigma}{\mathfrak{N}-J-1}, \quad j \in\{J+1, \ldots, \mathfrak{N}-1\} .
\end{aligned}
$$

In case of $2 m \Sigma \gg \omega$, we may use again the approximate form in Eq. (22), since in the relevant regime it leads to the correct leading order contribution as we have seen before, see Fig. 4. The prefactors (39) in (35) reduce then to an exponential that yields the following expression

$$
\begin{aligned}
& \mathcal{P}_{\mathfrak{N}} \simeq \exp \left(-\frac{4 m \Sigma}{\omega}\right) \\
& \times \exp \left(\frac{2 m^{2}}{E}\left[\Sigma \sqrt{1-\Sigma^{2}}+\arcsin (\Sigma)-\frac{\pi}{2}\right]\right) .
\end{aligned}
$$

Rescaling $2 m \Sigma \rightarrow \Sigma$ subsequently, the saddle point is simply given by $\Sigma_{\mathrm{sp}}=\varpi_{\mathrm{sp}} /(2 m)$. This is the same exponential factor as in (38) which remains unchanged for any $\mathfrak{N} \geq 1$. We conclude that similar as in the Sauter-like cases, the first order contribution in $\epsilon$ will be sufficient to approach the nonperturbative result. This is radically different from the ordinary Gaussian, i.e. $N=0$, which behaves nonperturbatively, since higher orders in $\epsilon$ turn out to be necessarily relevant [23].

\section{CONCLUSION}

We have discussed the Schwinger mechanism in the presence of an additional, uniformly oriented super Gaussian of integer order $4 N+2$. Using the worldline approach 
we have treated the resulting background nonperturbatively and showed that already for $N=2$ a much stronger dynamical enhancement applies in comparison to a weak contribution of Sauter type. More interestingly, we have shown that taking the limit $N \rightarrow \infty$, which corresponds to the usual rectangular potential barrier, results in the same leading order exponential factor as one finds for the bell shaped Lorentzian. Although both setups are highly distinct in Minkowski space, the found coincidence applies due to identical (effective) reflection points in the instanton plane which turn out to be the main regulator in this dynamical mechanism.

In addition, we have studied the impact of the weak super Gaussian in perturbation theory and found that in the limit $N \rightarrow \infty$ it shares the same higher order behaviour as Sauter-like pulses. We have argued that the leading order contribution in $\epsilon$ already approaches the nonperturbative result although a distinct pole structure, as one finds in the latter cases, is not present. Our results clearly demonstrate that tunnelling in such complex backgrounds can lead to nontrivial physics. Namely, the fact whether the superimposed weak super Gaussian behaves perturbatively or nonperturbatively depends on its microscopic details determined by the order parameter $N$.

\section{ACKNOWLEDGMENTS}

I thank Gudrid Moortgat-Pick for a careful reading of the manuscript. I acknowledge the support of the Colloborative Research Center SFB 676 of the DFG.

[1] J. S. Schwinger, Phys. Rev. 82, 664 (1951).

[2] D. Allor, T. D. Cohen, and D. A. McGady, Phys. Rev. D78, 096009 (2008), arXiv:0708.1471 [cond-mat.mes-hall].

[3] M. I. Katsnelson and G. E. Volovik, JETP Lett. 95, 411 (2012), [Pisma Zh. Eksp. Teor. Fiz.95,457(2012)], arXiv:1203.1578 [cond-mat.str-el].

[4] M. A. Zubkov, Pisma Zh. Eksp. Teor. Fiz. 95, 540 (2012), arXiv:1204.0138 [hep-ph].

[5] F. Fillion-Gourdeau and S. MacLean, Phys. Rev. B92, 035401 (2015).

[6] I. Akal, R. Egger, C. Müller, and S. Villalba-Chávez, Phys. Rev. D93, 116006 (2016), arXiv:1602.08310 [hep-ph].

[7] T. Oka and H. Aoki, Phys. Rev. Lett. 95, 137601 (2005).

[8] V. S. Popov, Sov. Phys. JETP. 34, 709 (1972), [Zh. Eksp. Teor. Fiz.61,1334(1971)].

[9] E. Brezin and C. Itzykson, Phys. Rev. D 2, 1191 (1970).

[10] G. V. Dunne and C. Schubert, Phys. Rev. D72, 105004 (2005), arXiv:hep-th/0507174 [hep-th].

[11] R. Schützhold, H. Gies, and G. Dunne, Phys. Rev. Lett. 101, 130404 (2008), arXiv:0807.0754 [hep-th].

[12] I. Akal, S. Villalba-Chávez, and C. Müller, Phys. Rev. D90, 113004 (2014), arXiv:1409.1806 [hep-ph].

[13] M. F. Linder, C. Schneider, J. Sicking, N. Szpak, and R. Schützhold, Phys. Rev. D92, 085009 (2015), arXiv:1505.05685 [hep-th].

[14] I. Akal and G. Moortgat-Pick, (2017), arXiv:1706.06447 [hep-th].

[15] I. Akal and G. Moortgat-Pick, (2017), arXiv:1710.04646 [hep-th].

[16] M. J. Strassler, Nucl. Phys. B385, 145 (1992), arXiv:hep-ph/9205205 [hep-ph].

[17] C. Schubert, Phys. Rept. 355, 73 (2001), arXiv:hep-th/0101036 [hep-th].

[18] G. V. Dunne (2004) pp. 445-522, arXiv:hep-th/0406216 [hep-th].

[19] I. K. Affleck, O. Alvarez, and N. S. Manton, Nucl. Phys. B197, 509 (1982).

[20] G. V. Dunne, Q.-h. Wang, H. Gies, and C. Schubert, Phys. Rev. D73, 065028 (2006), arXiv:hep-th/0602176 [hep-th].

[21] D. D. Dietrich and G. V. Dunne, J. Phys. A40, F825 (2007), arXiv:0706.4006 [hep-th].

[22] M. Holthaus, Physical review letters 69, 1596 (1992).

[23] G. Torgrimsson, C. Schneider, J. Oertel, and R. Schützhold, JHEP 06, 043 (2017), arXiv:1703.09203 [hep-th]. 